\title{
Chemical Design and Magnetic Ordering in Thin Layers of 2D Metal-Organic Frameworks (MOFs)
}

\author{
Javier López-Cabrelles, ${ }^{\perp}$ Samuel Mañas-Valero, ${ }^{\perp}$ Iñigo J. Vitórica-Yrezábal, Makars Š̌̌kins, Martin Lee, \\ Peter G. Steeneken, Herre S. J. van der Zant, Guillermo Mínguez Espallargas,* and Eugenio Coronado*
}

Cite This: J. Am. Chem. Soc. 2021, 143, 18502-18510

Read Online

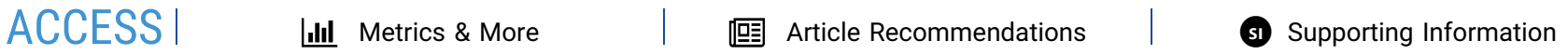

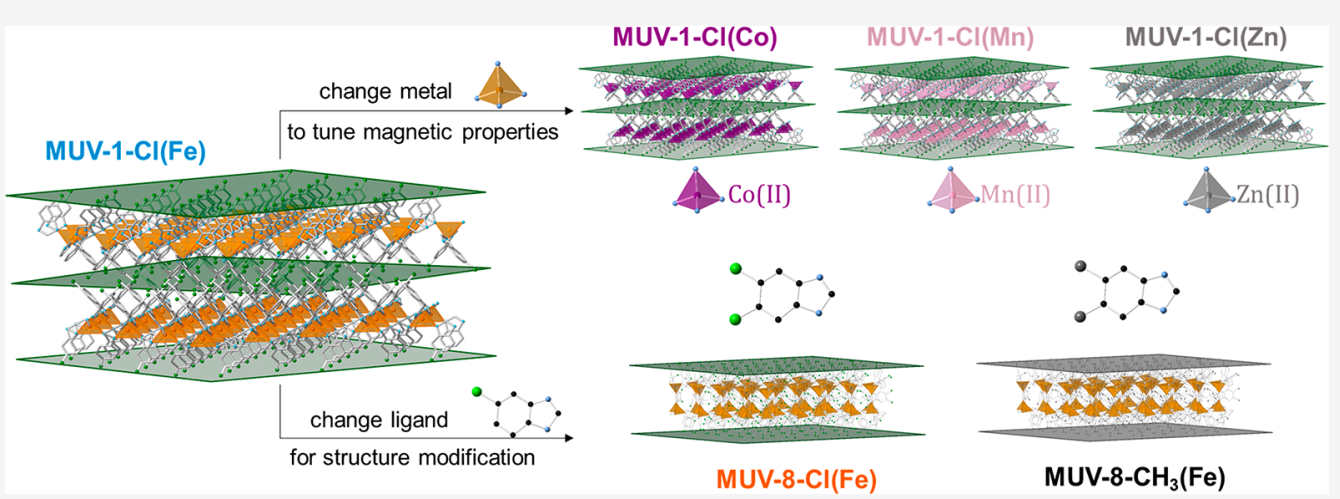

ABSTRACT: Through rational chemical design, and thanks to the hybrid nature of metal-organic frameworks (MOFs), it is possible to prepare molecule-based 2D magnetic materials stable at ambient conditions. Here, we illustrate the versatility of this approach by changing both the metallic nodes and the ligands in a family of layered MOFs that allows the tuning of their magnetic properties. Specifically, the reaction of benzimidazole-type ligands with different metal centers $\left(\mathrm{M}^{\mathrm{II}}=\mathrm{Fe}, \mathrm{Co}, \mathrm{Mn}, \mathrm{Zn}\right)$ in a solventfree synthesis produces a family of crystalline materials, denoted as MUV-1(M), which order antiferromagnetically with critical temperatures that depend on M. Furthermore, the incorporation of additional substituents in the ligand results in a novel system, denoted as MUV-8, formed by covalently bound magnetic double layers interconnected by van der Waals interactions, a topology that is very rare in the field of $2 \mathrm{D}$ materials and unprecedented for $2 \mathrm{D}$ magnets. These layered materials are robust enough to be mechanically exfoliated down to a few layers with large lateral dimensions. Finally, the robustness and crystallinity of these layered MOFs allow the fabrication of nanomechanical resonators that can be used to detect-through laser interferometry-the magnetic order in thin layers of these $2 \mathrm{D}$ molecule-based antiferromagnets.

\section{INTRODUCTION}

The isolation of atomically thin-single or few-layer-crystals has given rise to the emergence of the so-called twodimensional (2D) materials. These low-dimensional materials have shown a wide range of electronic and magnetic properties (from insulators to superconductors; from ferromagnets to antiferromagnets and quantum spin liquids) that can be affected by the dimensionality. ${ }^{1-5}$ Most of these materials derive from well-known layered inorganic materials, ${ }^{6}$ formed by covalently bound layers interconnected by weak van der Waals interactions, allowing the isolation of monolayers by exfoliation. A hot topic in this area deals with the isolation of monolayers of magnets with the aim of studying magnetism in the $2 \mathrm{D}$ limit and to integrate these layers in spintronic devices. ${ }^{7,8}$ The exploration of such experimental possibilities is hindered by the chemical instability in open air of the chosen inorganic compounds (mainly based on layered metal halides), thus limiting their manipulation and applicability. In fact, the first report on the magnetism of $\mathrm{CrI}_{3}$ down to the monolayer limit was published in $2017^{9}$ even though the discovery of its bulk properties has been known since $1959 .{ }^{10}$

Coordination chemistry can also offer a source of layered metal-organic materials which, in contrast to the inorganic analogs, are much more versatile from the point of view of the chemical design, chemical stability, and ease of manipulation in open air. However, the isolation of monolayers of these molecule-based magnets has been achieved in very few cases only. ${ }^{11}$ This may be due to the hybrid nature of the materials, which in most cases are formed by charged layers interleaved

Received: July 26, 2021

Published: November 1, 2021 


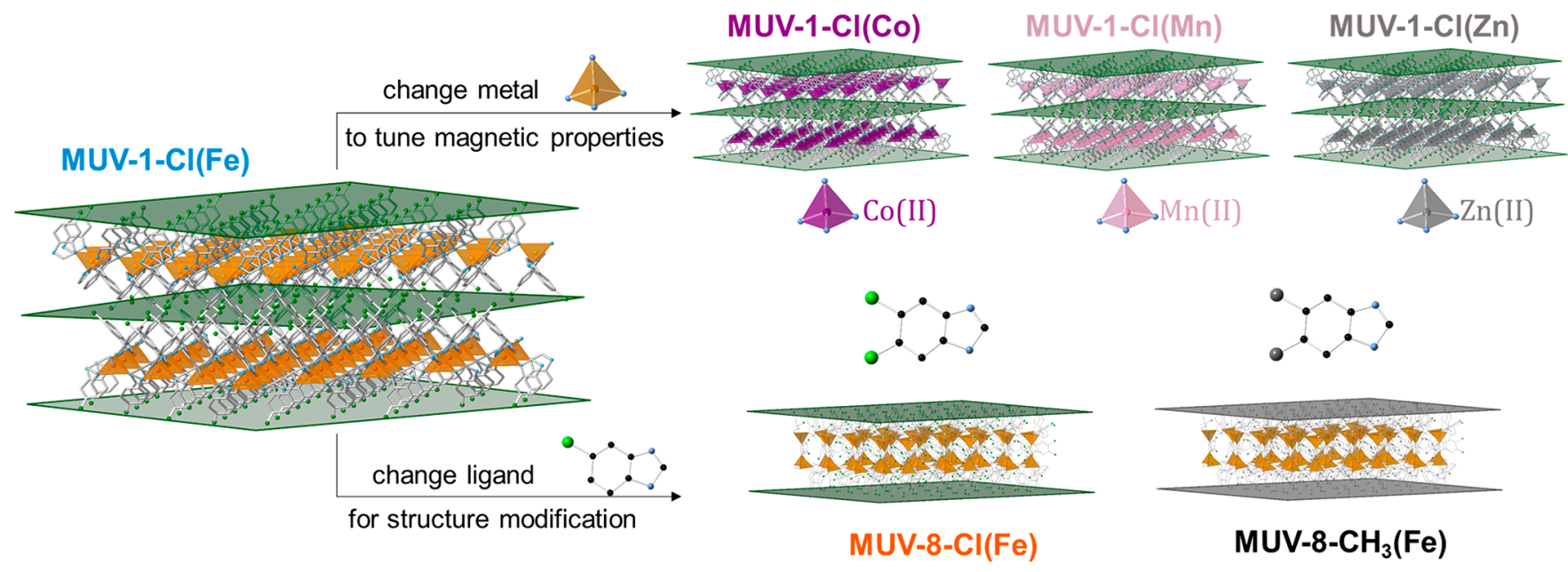

Figure 1. Chemical versatility of the MUV-1 system: it is possible to modify the magnetic properties by changing the metallic cation (upper part) and to induce structural changes by adding a second substituent to the benzimidazole ligand (bottom part), while keeping the layered morphology needed for a two-dimensional material.

by counterions. Hence, these layers are held together by electrostatic interactions and not by van der Waals interactions. This often results in fragile crystals of small sizes, which are very difficult to exfoliate using a micromechanical Scotch tape procedure. In fact, the first successful mechanical exfoliation of a layered magnet of this kind was reported by us in 2015 in the coordination polymer $\left[\mathrm{Fe}^{\mathrm{III}}\left(\mathrm{acac}_{2}\right.\right.$-trien $\left.)\right]\left[\mathrm{Mn}^{\mathrm{II}} \mathrm{Cr}^{\mathrm{III}}(\text { anilate })_{3}\right]$. $\left(\mathrm{CH}_{3} \mathrm{CN}\right)_{2}$, where anilate refers to dichloro- and dibromosubstituted anilate ligand. ${ }^{12}$ These compounds in bulk behave as ferrimagnets with a transition temperature, $T_{c}=11 \mathrm{~K}$. The structure of the magnetic layers consists of a hexagonal $2 \mathrm{D}$ anionic network formed by $\mathrm{Mn}^{2+}$ and $\mathrm{Cr}^{3+}$ ions linked through anilate ligands. In this case, and thanks to the large size of the hexagonal pores, the counterions $\left[\mathrm{Fe}^{\mathrm{III}}\left(\mathrm{acac}_{2} \text {-trien }\right)\right]^{+}$were inserted inside the magnetic framework instead of being in the interlamellar space. This feature facilitated the micromechanical exfoliation, leading to the isolation of magnetic layers with thicknesses down to $1.5 \mathrm{~nm}$ and lateral sizes on the order of hundreds of nanometers.

More recently, this result has been drastically improved by designing coordination compounds formed by neutral layers. ${ }^{13}$ To reach this goal we have exploited the chemical versatility provided by imidazolate-type ligands in the design of metal organic frameworks (MOFs) of various dimensionalities and properties. ${ }^{14-16}$ Thus, a isoreticular series of layered magnetic MOFs, composed by benzimidazole derivates and $\mathrm{Fe}(\mathrm{II})$ centers (MUV-1-X(Fe), with $\mathrm{X}=\mathrm{H}, \mathrm{Cl}, \mathrm{Br}, \mathrm{CH}_{3}$ or $\mathrm{NH}_{2}$ ) and behaving as spin-canted antiferromagnets with ordering Neel temperatures, $T_{\mathrm{N}}$, of $\sim 20 \mathrm{~K}$, has been synthesized by a solventfree method. ${ }^{13}$ In this series, monolayers have been isolated through micromechanical exfoliation, reaching high-quality crystalline flakes of micron size. On the other hand, we have also shown that these materials can be exfoliated in large amounts by using a liquid exfoliation method. ${ }^{17}$

Interestingly, the chemical versatility of this molecular approach has allowed us to functionalize the magnetic layer at will by changing the substituent $\mathrm{X}$ in the benzimidazole, while keeping its magnetic properties unchanged. ${ }^{13}$ Herein, we further exploit this versatility either by changing the metallic nodes, while maintaining the crystal structure, or by inserting a second substituent in the ligand, while retaining the layered morphology of the material (Figure 1). The first possibility provides the opportunity to tune the magnetic properties of the layers, while the second results in the isolation of covalently bound magnetic double-layers interconnected by van der Waals interactions, a topology that is very rare in the field of $2 \mathrm{D}$ materials and unprecedented for $2 \mathrm{D}$ magnets. In the second part of this work, these van der Waals antiferromagnets are mechanically exfoliated down to the atomically thin layers and we show that their magnetic ordering can be probed mechanically by nanomechanical resonators made of thin membranes of these insulating materials. Compared to ferromagnets, antiferromagnets exhibit high frequency magnon dynamics and are insensitive to external magnetic fields. Since most of them are insulators, they can present pure spin currents with no Joule heating, making them of potential interest for low-consumption spintronic memory devices. ${ }^{18}$ In the particular case of $2 \mathrm{D}$ antiferromagnets a larger variety of spin anisotropies, which can be optically controlled, have been experimentally shown. ${ }^{19}$

\section{RESULTS AND DISCUSSION}

Changing Metallic Nodes. The existence of different metal sources compatible with chemical vapor deposition and solvent-free methods permits the modification of the reaction previously described in ref 13 , where benzimidazole derivates ( HbimX, $\mathrm{X}=\mathrm{Cl}, \mathrm{Br}, \mathrm{CH}_{3}, \mathrm{H}, \mathrm{NH}_{2}$ ) and ferrocene were employed, using different metal cyclopentadienyls and other metal precursors (see details in Section S1). Obtaining isostructural materials changing the metal node is a challenge that leads to the modification of the properties of the materials, in the present case, the magnetic properties (Figure 1). Biscyclopentadienyl cobalt(II), bis (tetramethylcyclopentadienyl) manganese(II), bis(2,2,6,6-tetramethyl-3,5heptanedionato) zinc(II), and $\mathrm{ZnO}$ were used as metal precursors in a solvent-free method to synthesize isostructural compounds of MUV-1-Cl and MUV-1-H. The crystals obtained in the synthesis were characterized by single crystal $\mathrm{X}$-ray diffraction and/or powder X-ray diffraction (see Sections S2 and S3), obtaining three isostructural compounds of MUV1-Cl (Co, Mn, and Zn) and three of MUV-1-H (Co, Mn, and $\mathrm{Zn}$ ). The metal cation is located in the inner part of the layers in a distorted tetrahedral environment, connected by benzimidazole bridges, allowing magnetic exchange between 
the metal centers. The size of the crystals is smaller for the cobalt, manganese, and zinc compounds than for the previously reported iron analogue, but all of these materials keep the layered morphology (Figures 2 and S1-S3). These
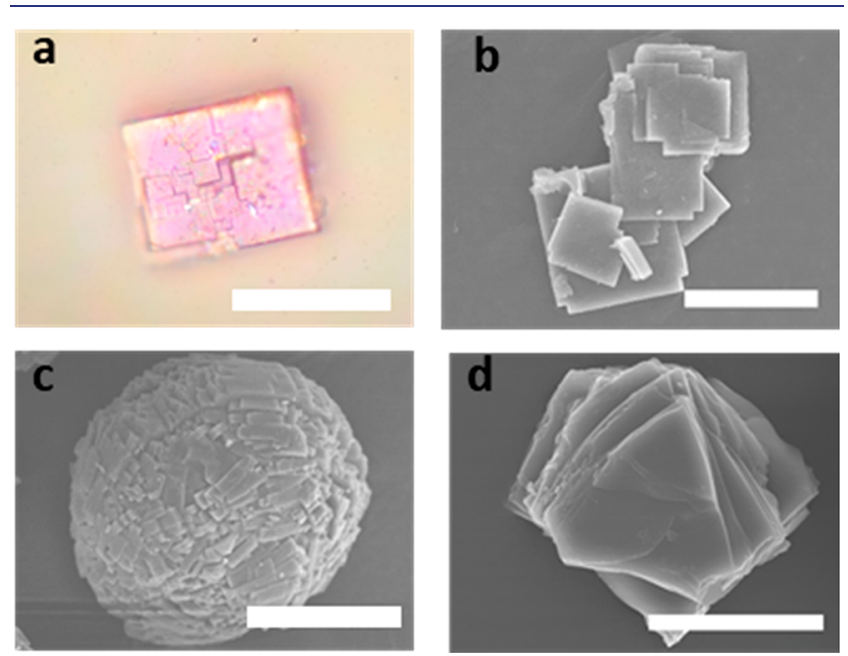

Figure 2. (a) Optical images for a crystal of MUV-1-Cl(Co). (b,c) SEM images of MUV-1-Cl(Co) and (d) MUV-8-Cl(Fe) showing the layered morphology. Note that $\mathrm{MUV}-1-\mathrm{Cl}(\mathrm{Co})$ presents spontaneous crystal aggregation with spherical uncommon morphologies. Scale bars are $10,10,20$, and $30 \mu \mathrm{m}$, respectively.

changes in the metallic nodes introduce changes in both the single-ion magnetic anisotropy and the intralayer exchange interactions that permit the tunability of the magnetic properties in these materials (see Magnetic Properties section).

Changing the Ligands. The molecular nature of the coordination polymers permits changes not only in the metal nodes but also in the ligand part (i.e., on the functional groups). In this case, a second substituent is added in the organic ligand adjacent to the 5 position where the initial substituent is present. Thus, two different ligands have been used, 5,6-dichlorobenzimidazole $\left(\mathrm{HbimCl}_{2}\right)$ and 5,6-dimethylbenzimidazole $\left(\mathrm{Hbim}\left(\mathrm{CH}_{3}\right)_{2}\right)$. Importantly, this change in the ligand does not affect the formation of a layered structure, and their reaction with ferrocene gives rise to layered coordination polymers, the so-called MUV-8- $\mathrm{Cl}(\mathrm{Fe})$ and $\mathrm{MUV}-8-\mathrm{CH}_{3}(\mathrm{Fe})$ (Figures 2 and S1). However, this second functional group induces a significant structural change resulting in unprecedented double layers of iron(II) centers arranged in distorted hexagons and linked through bridging benzimidazole ligands (Figure 3).

There are four crystallographically different iron centers in a single double layer of MUV-8 (Figure S2). These iron centers are connected by three bidentate ligands in the $a b$ plane, and a fourth bidentate ligand connecting two iron centers each in its respective layer (in the $c$ direction) (Figure 3biii). As a consequence, the resulting double layer in MUV-8 exhibits a thickness of $1.5 \mathrm{~nm}$, being formed by the sequence "ligand layer/Fe layer/ligand layer/Fe layer/ligand layer", which is large compared to the thickness of $1 \mathrm{~nm}$ in MUV-1 since that is formed just by one monolayer of $\mathrm{Fe}$ centers (sequence of "ligand layer/Fe layer/ligand layer"). The iron centers are located in very distorted tetrahedral sites, with $\mathrm{Fe}-\mathrm{N}$ lengths of 1.8-2.15 $\AA$, and connected by benzimidazolate bridges.

These connected layers are superposed in a similar arrangement to an $\mathrm{AB}$ stacking, with a small shift between

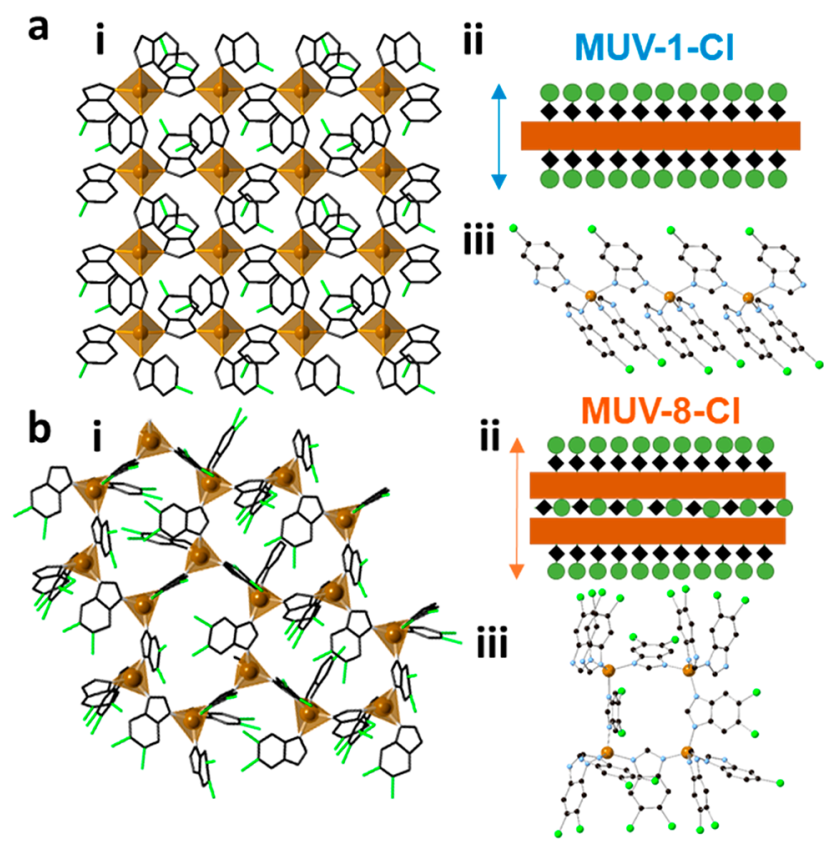

Figure 3. Comparison of the crystal structures of MUV-1-Cl (a) and MUV-8-Cl (b). Structure of a single layer (i) viewed along the $c$ axis ( $a b$ plane). Schematic representation of a single layer (ii), showing an increase thickness for the "double layer" of irons in the MUV-8-Cl (from 1 to $1.5 \mathrm{~nm}$ ). Coordination modes of the ligands (iii).

iron centers (Figure S2). The chlorine atoms belonging to the ligands placed in the $a b$ plane are pointing toward the surfaces and are weakly interacting through van der Waals forces like in the MUV-1-Cl case, while the bridging ligand connecting the two monolayers is located inside of the double layer. Moreover, in MUV-8-X, some ferrocene molecules are located between the layers, interacting weakly with the layers of the compound (Figure S3). These molecules cannot be removed with heat as can be seen by thermogravimetric analysis (Figure S6). The two-dimensional units are composed of covalently bound double layers held together through coordination bonds and interconnected by weak van der Waals interactions, instead of being formed by covalently bound monolayers. This novel structural arrangement opens the way to isolate unprecedented 2D magnetic networks using a coordination chemistry approach.

The layered morphology of the crystals (Figure 2) allows their delamination and the possibility to explore the $2 \mathrm{D}$ limit in these compounds. We have focused on the cobalt system, MUV-1-Cl(Co) and MUV-1-H(Co), and on MUV-8-Cl(Fe) and MUV-8- $\mathrm{CH}_{3}(\mathrm{Fe})$. Bulk crystals of these four systems were thinned-down by mechanical exfoliation as previously realized in MUV-1-Cl, ${ }^{13}$ yielding flakes with well-defined shapes (lateral dimensions of $>1 \mu \mathrm{m}$ ) and different thicknesses (from hundreds of nanometers down to a few nanometers). The obtained flakes are characterized by optical and atomic force (AFM) microscopies (Figure $4 \mathrm{a}$ and $4 \mathrm{~b}$, and Section S5) as well as Raman spectroscopy, in order to confirm their integrity and chemical composition. In the case of the novel structures of MUV-8, we have achieved thin-layer with a thickness of $6 \mathrm{~nm}$, which in this case corresponds to 3-4 monolayers. Importantly, we have been able to detect the Raman spectra of these ultrathin films (Figure 4c).

Magnetic Properties. The magnetic behavior of the polycrystalline bulk samples was investigated by SQUID 

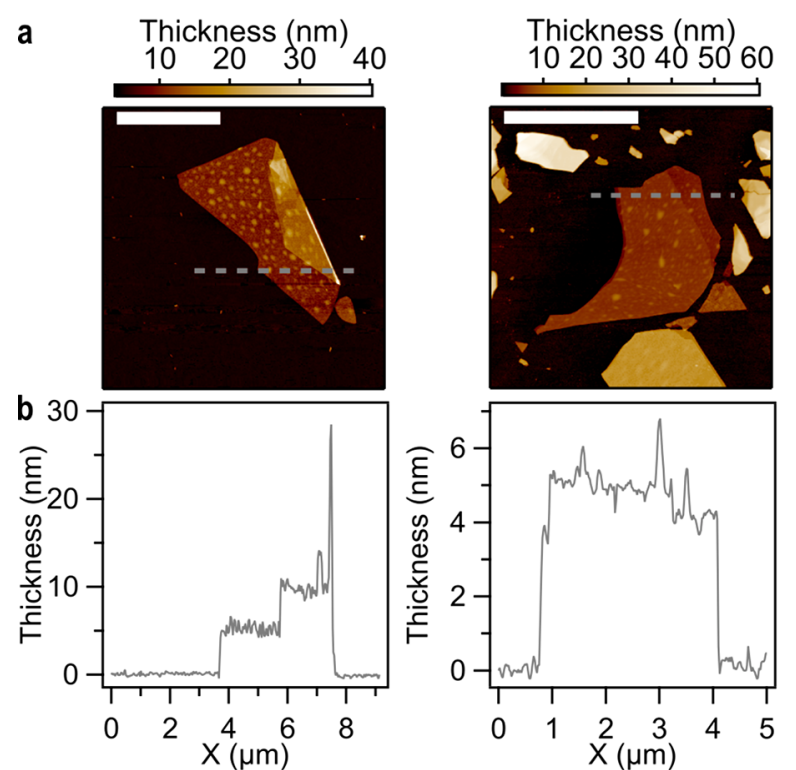

C
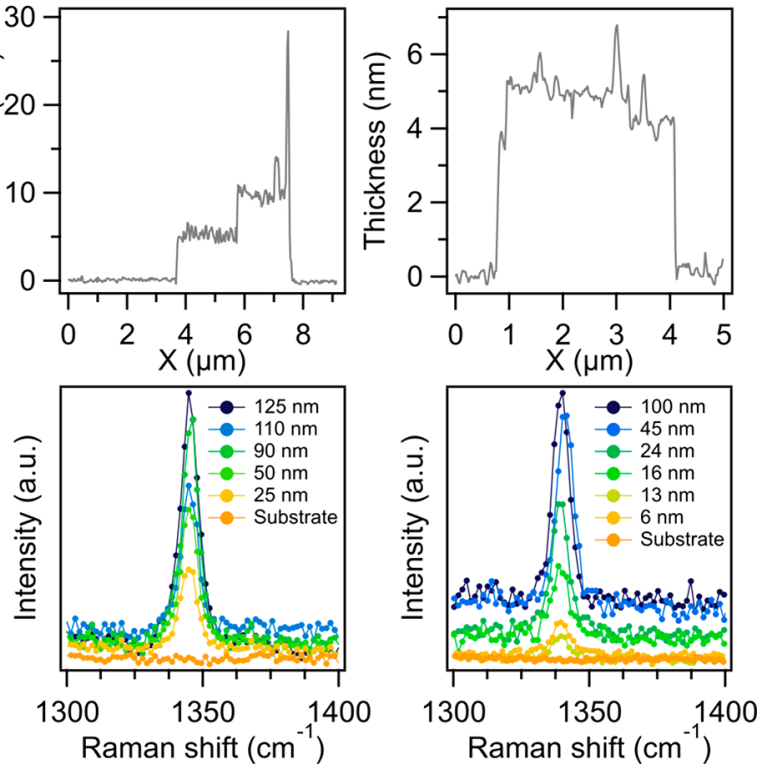

Figure 4. (a,b) AFM image and profile of a typical exfoliated MUV-1$\mathrm{Cl}(\mathrm{Co})$ (left) and MUV-8-Cl(Fe) flake (right). Scale bars are $5 \mu \mathrm{m}$. (c) Selected region of the Raman spectra of $\mathbf{M U V - 1 - C l ( C o ) ~ ( l e f t ) ~}$ and MUV-8-Cl(Fe) (right) flakes of different thicknesses.

measurements in the range $2-300 \mathrm{~K}$. Respective data can be found in the Supporting Information (SI) (see Section S4). In Figure 5 we plot the thermal variation of the susceptibility $(\chi)$ for the $\mathrm{Co}(\mathrm{II})$ and $\mathrm{Mn}$ (II) derivatives of MUV-1, as well as the $\mathrm{Fe}(\mathrm{II})$ derivative of MUV-8. In these compounds $\chi$ increases upon cooling down until a maximum or a levelling are reached at intermediate temperatures. This agrees with an antiferromagnetic coupling between the metal centers, also supported by the continuous decrease in the $\chi T$ product in this region (insets of the Figure). At lower temperatures, a sharp increase in $\chi$ is observed for the Co and Fe samples. This feature, together with the presence of a sharp out-of-phase component of the a.c. susceptibility signal, $\chi^{\prime \prime}$, at this temperature (Figure 5 , right) and magnetic hysteresis loops (Figure S18), is indicative of a transition toward antiferromagnetic order with spin canting at the critical temperature, $T_{\mathrm{N}}$. In contrast, in the $\mathrm{Mn}$ derivative, the maximum in $\chi$ is followed by a sharp decrease at lower temperature, which agrees with an antiferromagnetic ordered structure without canting (Figure 5 , left). This is corroborated by the absence of a $\chi^{\prime \prime}$ signal in the vicinity of $T_{N}$ (Figure 5, right). Such a difference may be related to the fully isotropic nature of the spin in the $\mathrm{Mn}$ derivative (with a ground term ${ }^{6} \mathrm{~A}_{1}$ ), while some magnetic anisotropy is expected for high-spin $\mathrm{Co}(\mathrm{II})$ and $\mathrm{Fe}(\mathrm{II})$ in tetrahedral sites (described by ${ }^{4} \mathrm{~A}_{2}$ and ${ }^{5} \mathrm{E}$ terms, respectively).

The critical temperatures in these antiferromagnets have been estimated from the temperature where $\chi^{\prime \prime}$ differs from 0 a
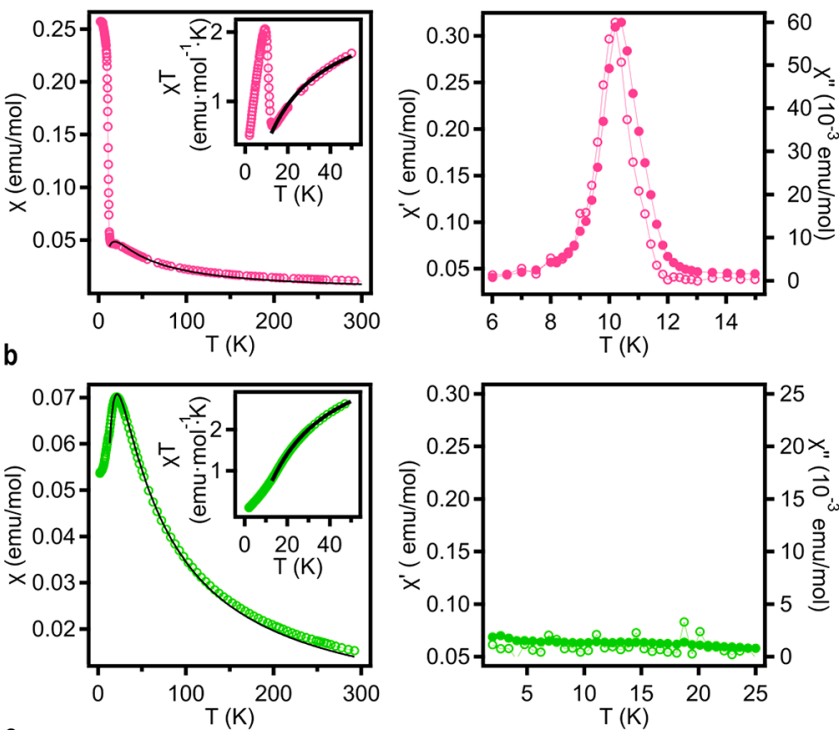

C
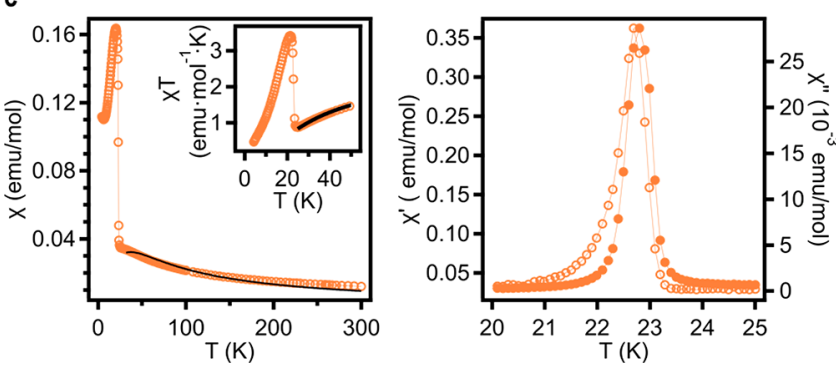

Figure 5. d.c. (left column) and a.c. (right column; in-phase and outof-phase components at $110 \mathrm{~Hz}$ are denoted with solid and open circles, respectively) magnetic susceptibilities for different MUV-1 and MUV-8 compounds. (a) MUV-1-Cl(Co), (b) MUV-1-H(Mn), (c) MUV-8-Cl(Fe). Solid black lines indicate the fit in the low dimensional regime to the expression for an antiferromagnetic square lattice (MUV-1) and the Curély formula for an antiferromagnetic hexagonal layer (MUV-8). ${ }^{21,22}$

in the case of systems with spin-canting (MUV-1(Co) and MUV-8(Fe)). In the case where the canting is absent (MUV$\mathbf{1}(\mathbf{M n})), T_{\mathrm{N}}$ is estimated, according to the Fisher criteria, ${ }^{20}$ by considering the maximum in $\partial(\chi T) / \partial T$. In this last system, a $T_{N}$ value of $14 \mathrm{~K}$ is derived, which is consistent with the EPR measurements that show important changes in the signal near this temperature (see Section S4.2). $T_{\mathrm{N}}$ values for all the materials are summarized in Table 1.

Finally, an estimate of the exchange parameters in these $2 \mathrm{D}$ antiferromagnetic networks is obtained by fitting the magnetic data to the corresponding theoretical models. To describe the behavior of MUV-1 derivatives, a model for a quadratic-layer Heisenberg antiferromagnet is used (Lines model; ${ }^{21}$ see SI). This model reproduces in particular the rounded maximum observed in $\chi$ in the $\mathrm{Mn}$ (II) derivatives (Figure $5 \mathrm{~b}$ ). The exchange parameters for $\mathbf{M U V - 8}(\mathbf{F e})$ are more difficult to extract since the magnetic network is very complex (see Figure S12). The structure involves two distorted hexagonal lattices, each one formed by two different exchange parameters, $J$ and $J_{0}$; these two layers are coupled by an additional exchange, $J_{\text {inter }}$. Since the in-plane distances are in the range 6.0-6.1 $\AA$, while the interplane ones are of $6.2 \AA$, we could expect the interplanar $\mathrm{Fe}-\mathrm{Fe}$ exchange interactions to be similar to the in-plane ones. There is no theoretical model available to 
Table 1. Magnetic Properties of the MUV-1 and MUV-8 Families ${ }^{a}$

\begin{tabular}{|c|c|c|c|c|c|c|}
\hline MUV & $S$ & $T_{\mathrm{N}}(\mathrm{K})$ & $J\left(\mathrm{~cm}^{-1}\right)$ & $g$ & $C\left(\mathrm{emu} \cdot \mathrm{mol}^{-1} \cdot \mathrm{K}\right)$ & $\Theta(\mathrm{K})$ \\
\hline MUV-1-Cl(Fe $)^{b}$ & 2 & 20.7 & $-22.9 \pm 0.4$ & $2.00 \pm 0.02$ & $3.635 \pm 0.006$ & $-80.6 \pm 0.4$ \\
\hline MUV-1-H $(\mathrm{Fe})^{b}$ & 2 & 20.0 & $-23.5 \pm 0.2$ & $1.98 \pm 0.02$ & $5.76 \pm 0.02$ & $-97.5 \pm 1.3$ \\
\hline MUV-1-Br $(\mathrm{Fe})^{b}$ & 2 & 20.0 & $-22.8 \pm 0.2$ & $2.0 \pm 0.2$ & $3.693 \pm 0.002$ & $-89.32 \pm 0.2$ \\
\hline MUV-1- $\mathrm{CH}_{3}(\mathrm{Fe})^{b}$ & 2 & 20.1 & $-22.6 \pm 0.4$ & $2.0 \pm 0.2$ & $5.13 \pm 0.03$ & $-132.0 \pm 1.9$ \\
\hline MUV-1-NH ${ }_{2}(\mathrm{Fe})^{b}$ & 2 & 21.2 & $-23.3 \pm 0.3$ & $2.0 \pm 0.2$ & $3.976 \pm 0.014$ & $-106.8 \pm 1.5$ \\
\hline MUV-1-Cl(Co) & $3 / 2$ & 11.6 & $-20.2 \pm 0.4$ & $2.3 \pm 0.2$ & $3.915 \pm 0.044$ & $-73 \pm 2$ \\
\hline MUV-1-H(Co) & $3 / 2$ & 12.4 & $-20.8 \pm 0.4$ & $2.2 \pm 0.2$ & $4.11 \pm 0.07$ & $-112 \pm 4$ \\
\hline MUV-1-Cl(Mn) & $5 / 2$ & 14.3 & $-10.7 \pm 0.2$ & $2.2 \pm 0.2$ & $6.60 \pm 0.05$ & $-65.9 \pm 1.5$ \\
\hline MUV-1-H(Mn) & $5 / 2$ & 14.8 & $-10.4 \pm 0.2$ & $2.02 \pm 0.10$ & $5.138 \pm 0.012$ & $-46.7 \pm 0.4$ \\
\hline MUV-8-Cl(Fe) & 2 & 23.2 & $-24.6 \pm 0.5$ & $2.0 \pm 0.2$ & $3.01 \pm 0.04$ & $-31.5 \pm 1.9$ \\
\hline MUV-8- $\mathrm{CH}_{3}(\mathrm{Fe})$ & 2 & 23.4 & $-25.2 \pm 0.5$ & $2.1 \pm 0.2$ & $4.80 \pm 0.06$ & $-121 \pm 3$ \\
\hline
\end{tabular}

${ }^{a} J$ and $g$ parameters are extracted by fitting the magnetic data to Lines ${ }^{21}$ and Curély ${ }^{22}$ models for quadratic and hexagonal exchange networks, respectively. The form of the exchange Hamiltonian is $\left(\mathcal{H}=-J \Sigma_{i, j} S_{i} \cdot S_{j}\right) \cdot{ }^{b} \mathbf{M U V}-\mathbf{1}(\mathbf{F e})$ data have been taken from ref 13.

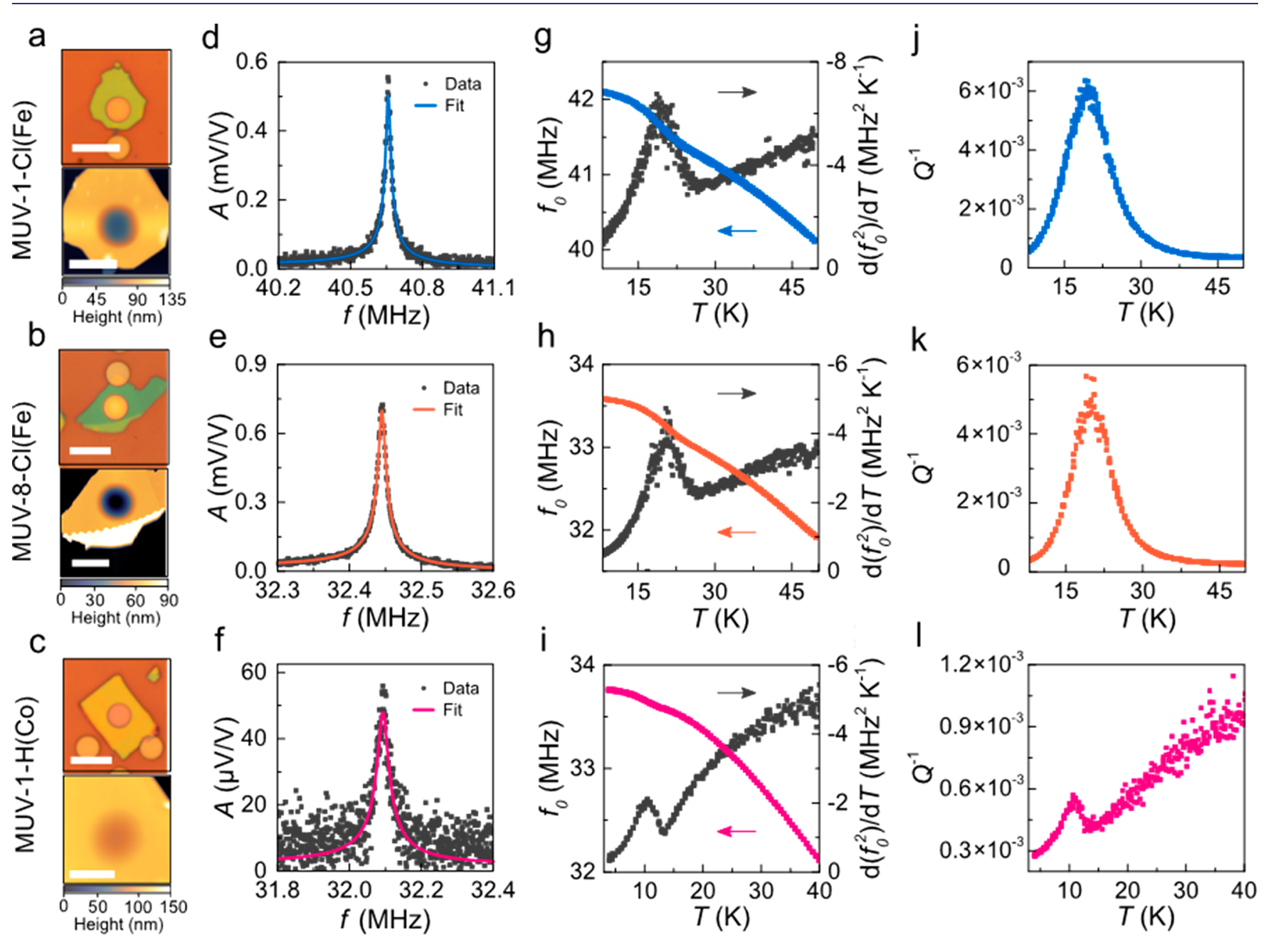

Figure 6. Mechanical resonances of thin MOF membranes. (a-c) MUV-1-Cl(Fe), MUV-8-Cl(Fe) and MUV-1-H(Co) membranes. (a-c) Top panels: Optical images. Bottom panels: Peak-force atomic force microscopy (AFM) images. (a) Scale bars: Top and bottom panel $-10 \mu \mathrm{m}$. Membrane thickness $t=87.7 \pm 0.7 \mathrm{~nm}$ and diameter $d=5 \mu \mathrm{m}$. (b) Scale bars: Top panel $-12 \mu \mathrm{m}$. Bottom panel $-6 \mu \mathrm{m}$. Membrane thickness $t=$ $65.2 \pm 1.3 \mathrm{~nm}$ and diameter $d=6 \mu \mathrm{m}$. (c) Scale bars: Top panel $-12 \mu \mathrm{m}$. Bottom panel $-6 \mu \mathrm{m}$. Membrane thickness $t=116.4 \pm 1.4 \mathrm{~nm}$ and diameter $d=6 \mu \mathrm{m}$. (d-f) Resonance peaks of the fundamental membrane mode at $40 \mathrm{~K}$. Colored lines - linear harmonic oscillator fit, black dots measured data. $(\mathrm{g}-\mathrm{i})$ Colored dots - resonance frequency $f_{0}$ as a function of temperature, black dots - temperature derivative of $f_{0}^{2}(T)$ as a function of temperature. (j-1) Mechanical dissipation $Q^{-1}$ as a function of temperature.

describe this network. The closest model available involves a single hexagonal lattice of classical spins isotropically coupled (Curély model, ${ }^{22}$ see Section S4). This model could provide a rough estimate of the exchange values in the MUV-8 material.
A close fit of the data is obtained using several sets of $J$ and $J_{0}$ values, indicating a strong correlation between them (see SI). In view of the structure, which shows similar $\mathrm{Fe}-\mathrm{Fe}$ distances (comprised in between 6.0 and $6.2 \AA$ ), we have assumed that 
both $J$ values should be similar and therefore we have set $J=J_{0}$. Using this model, an antiferromagnetic coupling $J=J_{0}=-25$ $\mathrm{cm}^{-1}$ is estimated, which is within the range of those obtained for MUV-1(Fe) compounds (see Table 1) and may support the above assumption. More precisely, it is slightly stronger $\left(-25 \mathrm{~cm}^{-1}\right.$ compared to $\left.-23 \mathrm{~cm}^{-1}\right)$, which is also in agreement with the higher values observed for $T_{\mathrm{N}}(23 \mathrm{~K}$ compared to $20 \mathrm{~K}$ ). As we can see in this table, the exchange coupling in all the compounds is antiferromagnetic. Interestingly, for a given metallic derivative, the magnetic properties remain unaltered, independent of the type of derivatization on the benzimidazole ligand. Still, these properties change upon changing the metal center (from Fe to Mn and Co for MUV-1, for example).

Magnetic Order by Nanomechanical Resonators. The detection of magnetic order in thin layers of insulating 2D materials is a challenging problem, as it is very difficult to sense such a small amount of material by conventional bulk characterization methods (magnetic or specific heat measurements). In fact, in these 2D materials magnetic ordering has been only detected recently in inorganic magnetic materials by performing Magneto-Optical Kerr Effect measurements at the nanoscale (nanoMOKE), ${ }^{9}$ transport measurements in van der Waals heterostructures, ${ }^{23-25}$ or $\mathrm{NV}$ (nitrogen vacancies) magnetometry. ${ }^{26}$ Indirect techniques, such as optical measurement of the second harmonic generation, have also been used to characterize thin layers of inorganic antiferromagnets. ${ }^{19,27}$ Recently, we have demonstrated that the specific heat can be extracted from measuring the temperature dependent nanomechanical resonance frequency of suspended 2D antiferromagnetic membranes. ${ }^{28}$ In particular, it can be shown that the resonance frequency of the fundamental membrane mode, $f_{0}$, and the quality factor, $Q$ of a nanomechanical resonator are related to the specific heat, $c_{\mathrm{v}}$, by the following relations: $c_{\mathrm{v}}(T)$ $\propto \mathrm{d}\left(f_{0}^{2}(T)\right) / \mathrm{d} T$ and $Q^{-1} \propto c_{v}(T) \times T$. These relations have been used to prove the antiferromagnetic order in the inorganic layers $\mathrm{FePS}_{3}, \mathrm{MnPS}_{3}$, and $\mathrm{NiPS}_{3}{ }^{28}$ However, the larger fragility of metal-organic materials to lasers has prevented so far detection of the magnetic order in thin layers of these molecular magnets. In our previous work, we demonstrated that the MUV-1 family is robust enough to fabricate mechanical resonators and to measure its mechanical properties through laser interferometry. ${ }^{13}$ Taking advantage of this feature-uncommon for a molecular material-we will explore here the possibility to detect magnetic order in these molecular layers using this nanomechanical technique.

We exfoliate flakes of MUV-1-Cl(Fe), MUV-8-Cl(Fe), and MUV-1-H(Co) and transfer these on top of circular cavities (diameter $d=5-6 \mu \mathrm{m}$ ) etched in a $\mathrm{SiO}_{2} / \mathrm{Si}$ substrate using deterministic dry viscoelastic stamping to form freestanding nanodrums (see Figure $6 a-c$ ). ${ }^{29}$ Due to the large flexibility, low estimated Young's modulus and, thus, low bending rigidity of these MOF sheets, the suspended flakes behave as membranes even at relatively large thicknesses for a given range of cavity diameters. ${ }^{13}$ The samples are placed in a dry cryostat and cooled down to temperatures of $4 \mathrm{~K}$ at a pressure below $10^{-6} \mathrm{mbar}$. Temperature-dependent mechanical properties of the nanodrums are then investigated using laser interferometry ${ }^{28}$ from 4 to $50 \mathrm{~K}$ in the absence of an external magnetic field (see Experimental Section). Figure 6d-f shows resonances of the fundamental membrane mode at $40 \mathrm{~K}$ for MUV-1-Cl(Fe), MUV-8-Cl(Fe), and MUV-1-H(Co), respectively (black solid dots).
We fit the measured resonance peaks to a linear harmonic oscillator model (colored solid lines) and obtain $f_{0}$ and $Q$. Substantially large $Q$ factors ranging from 1000 to 3500 and high resonance frequencies of $32.1-40.67 \mathrm{MHz}$ at $40 \mathrm{~K}$ indicate a high tension in the membranes due to a buildup of the thermal strain. Since the thermal strain in the membranes is related to the thermal expansion coefficient and thus to the specific heat $c_{\mathrm{v}}$ of the material, we will check if any anomaly is observed in $f_{0}(T)$, related to a phase change. ${ }^{21}$ In Figure $6 \mathrm{~g}-\mathrm{i}$ we plot $f_{0}$ (colored filled dots) and the corresponding temperature derivative of $f^{2}$ (black filled dots) for all three compounds. The phase transition-related anomaly is well visible as a kink in $f_{0}(T)$ and is even more pronounced in $\mathrm{d}\left(f^{2}{ }_{0}\right.$ $(T)) / \mathrm{d} T$, which shows a peak at low temperatures that is associated with $T_{\mathrm{N}}$. As can be seen in Figure 7, the transition

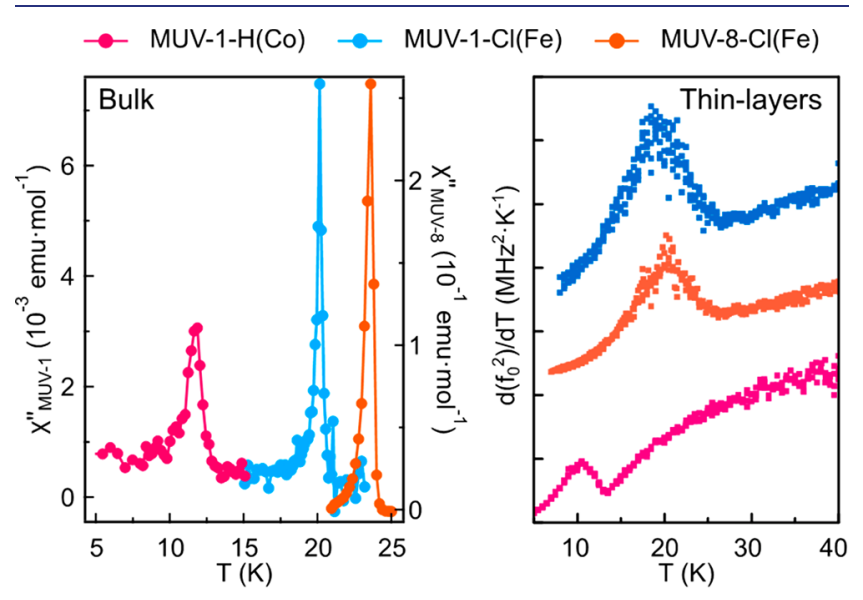

Figure 7. Comparison of the out-of-phase a.c. susceptibility signals for bulk systems (left) and the derivative of $f_{0}^{2}(T)$ as a function of temperature for thin layers (right).

temperatures for the thin layers (thickness in the range 65 to $120 \mathrm{~nm}$ ), extracted from these peaks, are in close agreement with the values determined in Table 1 for the bulk counterparts by a.c. magnetic measurements (MUV-1-Cl(Fe): $19.5 \pm 1.0 \mathrm{~K}$, compared to $20.7 \mathrm{~K} ; \mathrm{MUV}-8-\mathrm{Cl}(\mathrm{Fe}): 20.5 \pm 1.0$ $\mathrm{K}$, compared to $23.2 \mathrm{~K}$; MUV-1-H(Co): $11.0 \pm 1.0 \mathrm{~K}$, compared to $12.4 \mathrm{~K})$. The mechanical dissipation $Q^{-1}(T)$ also exhibits a local maximum near $T_{\mathrm{N}}$, as displayed in Figure $6 \mathrm{j}-\mathrm{l}$, that can be related to thermoelastic or other more intricate magnetomotive damping mechanism. ${ }^{28}$

\section{CONCLUSIONS}

At the intersection between the fields of molecular magnetism and $2 \mathrm{D}$ materials, we have exploited the chemical design of layered magnetic coordination polymers based on benzimidazole derivatives with the aim of producing novel $2 \mathrm{D}$ molecularbased magnets with novel topologies and tunable magnetic properties. Thus, using a solvent-free synthetic route, we have prepared an isoreticular series of layered materials formed by van der Waals layers with a square $2 \mathrm{D}$ magnetic lattice, in which the magnetic properties can be tuned by changing the metallic nodes (from $\mathrm{Fe}(\mathrm{II})$ to $\mathrm{Co}(\mathrm{II})$ and $\mathrm{Mn}(\mathrm{II})$ ), while preserving the same crystal structure. In addition, by modifying the organic part (from monosubstituted to disubstituted benzimidazole), it has been possible to change the crystal structure and the magnetic topology from a square lattice to a distorted hexagonal one. 
Interestingly, the very weak van der Waals forces between the layers has allowed us to isolate atomically thin layers of micrometer size flakes using a micromechanical exfoliation method. This result can be highly relevant in the field of $2 \mathrm{D}$ materials: First, it can offer an alternative way to that provided by solid-state chemistry for the preparation of robust $2 \mathrm{D}$ magnets. In fact, the coordination chemistry approach has afforded the isolation of $2 \mathrm{D}$ magnetic materials relatively stable in open air, in sharp contrast to the few reported examples of 2D inorganic magnets. Second, the chemical versatility of the method allows novel magnetic topologies to be obtained in two dimensions. The most relevant result in this context has been the isolation of a double layer antiferromagnet exhibiting an unprecedented topology, which is based on the assembly of two hexagonal monolayers through coordination bonds. Third, the employed solvent-free methodology is compatible with chemical vapor deposition techniques, thus allowing the future scalability of these 2D magnetic MOFs.

Nanomechanical resonators made from the MOFs have been used to detect the magnetic order in thin layers of these 2D molecular-based antiferromagnets. Due to the strong coupling between mechanical motion and magnetic order, this technique has provided additional confirmation of the critical temperatures in these thin layer systems. The use of this nanomechanical approach for detecting magnetic order in molecular materials is believed to be limited by their inherent instability to the exposure of the laser beam, which can damage the suspended membranes. Still, the layered MOFs studied have shown to be stable enough to be measured using this technique. In the present case, this has allowed the study of the magnetic order in membranes with layer thickness down to 65 $\mathrm{nm}$, although this thickness is still far from the 2D limit (1-2 $\mathrm{nm})$. More robust and thinner membranes will be prepared in the future taking advantage of the possibility of fabricating van der Waals heterostructures using a deterministic method. ${ }^{30}$ For example, by combining ultrathin layers of these MOFs with atomically thin layers of an inorganic material (h-BN layer, for instance), we expect to overcome this limitation. This will open interesting possibilities in $2 \mathrm{D}$ physics. Also, the tuning of $T_{\mathrm{N}}$ with the dimensionality of the system (number of magnetic layers), or with the strain of the membrane induced by an external stimulus (like an electrostatic gate voltage), ${ }^{31}$ could in future be realized.

\section{EXPERIMENTAL SECTION}

All reagents were commercially available and were used without further purification.

Synthesis of MUV-1-X(M"). A metallic source $(0.16 \mathrm{mmol})$ and benzimidazole derivate $(0.34 \mathrm{mmol})$ were combined and sealed under vacuum in a layering tube (4 $\mathrm{mm}$ diameter). The mixture was heated at $150{ }^{\circ} \mathrm{C}$ for 4 days to obtain crystals suitable for X-ray single-crystal diffraction. The product was allowed to cool to room temperature, and the layering tube was then opened. The unreacted precursors were extracted with acetonitrile and benzene, and the main compound was isolated as crystals (yield 60\%). Phase purity was established by $\mathrm{X}$-ray powder diffraction.

Synthesis of MUV-8-X(Fe). Ferrocene $(30 \mathrm{mg}, 0.16 \mathrm{mmol})$ and 5,6-dichlorobenzimidazole $(64 \mathrm{mg}, 0.34 \mathrm{mmol})$ or 5,6-dimethylbenzimidazole $(50 \mathrm{mg}, 0.34 \mathrm{mmol})$ were combined and sealed under vacuum in a layering tube ( $4 \mathrm{~mm}$ diameter). The mixture was heated at $250{ }^{\circ} \mathrm{C}$ for 3 days to obtain colorless crystals suitable for X-ray single-crystal diffraction. The product was allowed to cool to room temperature, and the layering tube was then opened. The unreacted precursors were extracted with acetonitrile and benzene, and the main compound was isolated as colorless crystals (yield 60\%). Phase purity was established by X-ray powder diffraction.

Single Crystal X-ray Diffraction. X-ray data for compounds MUV-1-H(Co) and MUV-8-Cl(Fe) were collected at a temperature of $120 \mathrm{~K}$ using a Mo-k $\alpha$ radiation on a Rigaku Supernova diffractometer equipped with an Oxford Cryosystems nitrogen flow gas system. X-ray data for compound MUV-1-Cl(Mn) were collected at a temperature of $100 \mathrm{~K}$ using a $\mathrm{Cu}-\mathrm{k} \alpha$ radiation on a Rigaku FR-X diffractometer equipped with an Oxford Cryosystems nitrogen flow gas system. Details on the crystal structure determination and refinements can be found in Section S2 of the Supporting Information.

Magnetic Properties. Variable-temperature $(2-300 \mathrm{~K})$ direct current (d.c.) magnetic susceptibility measurements were carried out in an applied field of $1.0 \mathrm{kOe}$, and variable field magnetization measurements up to $\pm 5 \mathrm{~T}$, at $2.0 \mathrm{~K}$. The susceptibility data were corrected from the diamagnetic contributions as deduced by using Pascal's constant tables. Variable-temperature (16-23 K) alternating current (ac) magnetic susceptibility measurements in a $\pm 4.0 \mathrm{G}$ oscillating field at frequencies in the range $1-997 \mathrm{~Hz}$ were carried out in a zero d.c. field. All the measurements were performed with a SQUID magnetometer (Quantum Design MPMS-XL-5 and MPMSXL-7).

Laser Interferometry. The motion of the nanodrums was measured using a laser interferometry setup, similar to the one reported in ref 28. The sample is mounted on an xyz piezomotive nanopositioning stage inside the chamber of a Montana Cryostation s50 dry optical cryostat. A blue diode laser $(\lambda=405 \mathrm{~nm})$, which is power-modulated by a Vector Network Analyzer (VNA), is focused at the center of the membrane and used to optothermally excite the membrane into motion at a given frequency. A red $\mathrm{He}-\mathrm{Ne}$ laser $(\lambda=$ $632 \mathrm{~nm}$ ) is used to read out the vibrations of the nanodrum, which are analyzed using a homodyne detection scheme and processed by the VNA. The laser spot diameter is in the order of $1 \mu \mathrm{m}$. It is checked that the resonance frequency changes due to laser heating are insignificant for all membranes.

Peak-Force AFM. All peak-force AFM data are acquired at a constant $50 \mathrm{nN}$ of applied peak force using a cantilever with a spring constant $k=22.8 \mathrm{~N} \mathrm{~m}^{-1}$ on Bruker Dimension FastScan AFM. The thickness of the sample is estimated taking the average of 3 to 5 profile scans.

\section{ASSOCIATED CONTENT}

\section{Supporting Information}

The Supporting Information is available free of charge at https://pubs.acs.org/doi/10.1021/jacs.1c07802.

Additional experimental details, materials, and methods (PDF)

\section{Accession Codes}

CCDC 2068293-2068295 contain the supplementary crystallographic data for this paper. These data can be obtained free of charge via www.ccdc.cam.ac.uk/data_request/cif, or by emailing data_request@ccdc.cam.ac.uk, or by contacting The Cambridge Crystallographic Data Centre, 12 Union Road, Cambridge CB2 1EZ, UK; fax: +44 1223336033.

\section{AUTHOR INFORMATION}

\section{Corresponding Authors}

Guillermo Mínguez Espallargas - Instituto de Ciencia

Molecular (ICMol), Universidad de Valencia, 46980

Paterna, Spain; 이이.org/0000-0001-7855-1003;

Email: guillermo.minguez@uv.es

Eugenio Coronado - Instituto de Ciencia Molecular (ICMol), Universidad de Valencia, 46980 Paterna, Spain;

(1) orcid.org/0000-0002-1848-8791;

Email: eugenio.coronado@uv.es 


\section{Authors}

Javier López-Cabrelles - Instituto de Ciencia Molecular (ICMol), Universidad de Valencia, 46980 Paterna, Spain; (1) orcid.org/0000-0001-7443-4635

Samuel Mañas-Valero - Instituto de Ciencia Molecular (ICMol), Universidad de Valencia, 46980 Paterna, Spain; (1) orcid.org/0000-0001-6319-9238

Iñigo J. Vitórica-Yrezábal - School of Chemistry, University of Manchester, Manchester M13 9PL, U.K.

Makars Siškins - Kavli Institute of Nanoscience, Delft University of Technology, 2628 CJ Delft, The Netherlands; (1) orcid.org/0000-0003-4295-2221

Martin Lee - Kavli Institute of Nanoscience, Delft University of Technology, 2628 CJ Delft, The Netherlands

Peter G. Steeneken - Kavli Institute of Nanoscience, Delft University of Technology, 2628 CJ Delft, The Netherlands; Department of Precision and Microsystems Engineering, Delft University of Technology, 2628 CD Delft, The Netherlands

Herre S. J. van der Zant - Kavli Institute of Nanoscience, Delft University of Technology, 2628 CJ Delft, The Netherlands; (1) orcid.org/0000-0002-5385-0282

Complete contact information is available at: https://pubs.acs.org/10.1021/jacs.1c07802

\section{Author Contributions \\ ${ }^{\perp}$ J.L.-C. and S.M.-V. contributed equally.}

Notes

The authors declare no competing financial interest.

\section{ACKNOWLEDGMENTS}

Financial support from the EU (ERC Advanced Grant MOL2D 788222, ERC Consolidator Grant S-CAGE 724681 and FET-OPEN SINFONIA 964396), the Spanish MICINN (PID2020-117177GB-I00, PID2020-117152RB-I00 cofinanced by FEDER, and Unit of Excellence María de Maeztu CEX2019-000919-M), the Generalitat Valenciana (PROMETEO program, iDiFEDER/2018/061 and iDiFEDER/2020/ 063) is gratefully acknowledged. J.L.-C. acknowledges the Universitat de València for an "Atracció de Talent" fellowship. M.S., M.L., P.G.S., and H.S.J.v.d.Z. acknowledge funding from the European Union's Horizon 2020 research and innovation program under Grant Agreement Number 881603.

\section{REFERENCES}

(1) Ferrari, A. C.; Bonaccorso, F.; Fal'ko, V.; Novoselov, K. S.; Roche, S.; Bøggild, P.; Borini, S.; Koppens, F. H. L.; Palermo, V.; Pugno, N.; Garrido, J. A.; Sordan, R.; Bianco, A.; Ballerini, L.; Prato, M.; Lidorikis, E.; Kivioja, J.; Marinelli, C.; Ryhänen, T.; Morpurgo, A.; Coleman, J. N.; Nicolosi, V.; Colombo, L.; Fert, A.; GarciaHernandez, M.; Bachtold, A.; Schneider, G. F.; Guinea, F.; Dekker, C.; Barbone, M.; Sun, Z.; Galiotis, C.; Grigorenko, A. N.; Konstantatos, G.; Kis, A.; Katsnelson, M.; Vandersypen, L.; Loiseau, A.; Morandi, V.; Neumaier, D.; Treossi, E.; Pellegrini, V.; Polini, M.; Tredicucci, A.; Williams, G. M.; Hee Hong, B.; Ahn, J.-H.; Min Kim, J.; Zirath, H.; van Wees, B. J.; van der Zant, H.; Occhipinti, L.; Di Matteo, A.; Kinloch, I. A.; Seyller, T.; Quesnel, E.; Feng, X.; Teo, K.; Rupesinghe, N.; Hakonen, P.; Neil, S. R. T.; Tannock, Q.; Löfwander, T.; Kinaret, J. Science and Technology Roadmap for Graphene, Related Two-Dimensional Crystals, and Hybrid Systems. Nanoscale 2015, 7 (11), 4598-4810.

(2) Novoselov, K. S.; Mishchenko, A.; Carvalho, A.; Castro Neto, A. H. 2D Materials and van Der Waals Heterostructures. Science 2016, 353 (6298), aac9439-aac9439.
(3) Gibertini, M.; Koperski, M.; Morpurgo, A. F.; Novoselov, K. S. Magnetic 2D Materials and Heterostructures. Nat. Nanotechnol. 2019, 14 (May), 408.

(4) Boix-Constant, C.; Mañas-Valero, S.; Córdoba, R.; Baldoví, J. J.; Rubio, A.; Coronado, E. Out-of-Plane Transport of $1 \mathrm{~T}-\mathrm{TaS}_{2}$ /Graphene-Based van Der Waals Heterostructures. ACS Nano 2021, $15,11898$.

(5) Manzeli, S.; Ovchinnikov, D.; Pasquier, D.; Yazyev, O. V.; Kis, A. 2D Transition Metal Dichalcogenides. Nature Reviews Materials 2017, DOI: $10.1038 /$ natrevmats.2017.33.

(6) Molina-Mendoza, A. J.; Giovanelli, E.; Paz, W. S.; Ninõ, M. A.; Island, J. O.; Evangeli, C.; Aballe, L.; Foerster, M.; Van Der Zant, H. S. J.; Rubio-Bollinger, G.; Agrait, N.; Palacios, J. J.; Pérez, E. M.; Castellanos-Gomez, A. Franckeite as a Naturally Occurring van Der Waals Heterostructure. Nat. Commun. 2017, 8. DOI: 10.1038/ ncomms 14409 .

(7) Ahn, E. C. 2D Materials for Spintronic Devices. npj 2D Mater. Appl. 2020, 4 (1). DOI: 10.1038/s41699-020-0152-0.

(8) Sierra, J. F.; Fabian, J.; Kawakami, R. K.; Roche, S.; Valenzuela, S. O. Van der Waals Heterostructures for Spintronics and OptoSpintronics. Nat. Nanotechnol. 2021, 16, 856-868.

(9) Huang, B.; Clark, G.; Navarro-Moratalla, E.; Klein, D. R.; Cheng, R.; Seyler, K. L.; Zhong, Di.; Schmidgall, E.; McGuire, M. A.; Cobden, D. H.; Yao, W.; Xiao, D.; Jarillo-Herrero, P.; Xu, X. Layer-Dependent Ferromagnetism in a van Der Waals Crystal down to the Monolayer Limit. Nature 2017, 546 (7657), 270-273.

(10) Hansen, W. N. Some Magnetic Properties of the Chromium (III) Halides at 4.2 K. J. Appl. Phys. 1959, 30 (4), S304-S305.

(11) Coronado, E. Molecular Magnetism: From Chemical Design to Spin Control in Molecules, Materials and Devices. Nat. Rev. Mater. 2020, 5, 87.

(12) Abhervé, A.; Mañas-Valero, S.; Clemente-León, M.; Coronado, E. Graphene Related Magnetic Materials: Micromechanical Exfoliation of 2D Layered Magnets Based on Bimetallic Anilate Complexes with Inserted $\left[\mathrm{Fe}^{\mathrm{III}}\left(\mathrm{Acac}_{2}-\mathrm{Trien}\right)\right]^{+}$and $\left[\mathrm{Fe}^{\mathrm{III}}\left(\mathrm{Sal}_{2}-\mathrm{Trien}\right)\right]^{+}$Molecules. Chem. Sci. 2015, 6 (8), 4665-4673.

(13) López-Cabrelles, J.; Mañas-Valero, S.; Vitórica-Yrezábal, I. J.; Bereciartua, P. J.; Rodríguez-Velamazán, J. A.; Waerenborgh, J. C.; Vieira, B. J. C.; Davidovikj, D.; Steeneken, P. G.; van der Zant, H. S. J.; Mínguez Espallargas, G.; Coronado, E. Isoreticular Two-Dimensional Magnetic Coordination Polymers Prepared through PreSynthetic Ligand Functionalization. Nat. Chem. 2018, 10 (10), 1001-1007.

(14) Park, K. S.; Ni, Z.; Côté, A. P.; Choi, J. Y.; Huang, R.; UribeRomo, F. J.; Chae, H. K.; O’Keeffe, M.; Yaghi, O. M. Exceptional Chemical and Thermal Stability of Zeolitic Imidazolate Frameworks. Proc. Natl. Acad. Sci. U. S. A. 2006, 103 (27), 10186-10191.

(15) Banerjee, R.; Phan, A.; Wang, B.; Knobler, C.; Furukawa, H.; O'Keeffe, M.; Yaghi, O. M. High-Throughput Synthesis of Zeolitic Imidazolate Frameworks and Application to $\mathrm{CO}_{2}$ Capture. Science 2008, 319 (5865), 939-943.

(16) López-Cabrelles, J.; Romero, J.; Abellán, G.; Giménez-Marqués, M.; Palomino, M.; Valencia, S.; Rey, F.; Mínguez Espallargas, G. Solvent-Free Synthesis of ZIFs: A Route toward the Elusive Fe(II) Analogue of ZIF-8. J. Am. Chem. Soc. 2019, 141 (17), 7173-7180.

(17) León-Alcaide, L.; López-Cabrelles, J.; Mínguez Espallargas, G.; Coronado, E. 2D Magnetic MOFs with Micron-Lateral Size by Liquid Exfoliation. Chem. Commun. 2020, 56 (55), 7657-7660.

(18) Jungwirth, T.; Marti, X.; Wadley, P.; Wunderlich, J. Antiferromagnetic Spintronics. Nat. Nanotechnol. 2016, 11 (3), 231-241.

(19) Afanasiev, D.; Hortensius, J. R.; Matthiesen, M.; Mañas-Valero, S.; Šškkins, M.; Lee, M.; Lesne, E.; van der Zant, H. S. J.; Steeneken, P. G.; Ivanov, B. A.; Coronado, E.; Caviglia, A. D. Controlling the Anisotropy of a van Der Waals Antiferromagnet with Light. Sci. Adv. 2021, 7 (23), eabf3096.

(20) Fisher, M. E. Relation between the Specific Heat and Susceptibility of an Antiferromagnet. Philos. Mag. 1962, 7 (82), $1731-1743$ 
(21) Lines, M. E. The Quadratic-Layer Antiferromagnet. J. Phys. Chem. Solids 1970, 31 (1), 101-116.

(22) Curély, J.; Lloret, F.; Julve, M. Thermodynamics of the TwoDimensional Heisenberg Classical Honeycomb Lattice. Phys. Rev. B: Condens. Matter Mater. Phys. 1998, 58 (17), 11465-11483.

(23) Klein, D. R.; MacNeill, D.; Lado, J. L.; Soriano, D.; NavarroMoratalla, E.; Watanabe, K.; Taniguchi, T.; Manni, S.; Canfield, P.; Fernández-Rossier, J.; Jarillo-Herrero, P. Probing Magnetism in 2D van Der Waals Crystalline Insulators via Electron Tunneling. Science 2018, 360 (6394), 1218-1222.

(24) Long, G.; Henck, H.; Gibertini, M.; Dumcenco, D.; Wang, Z.; Taniguchi, T.; Watanabe, K.; Giannini, E.; Morpurgo, A. F. Persistence of Magnetism in Atomically Thin $\mathrm{MnPS}_{3}$ Crystals. Nano Lett. 2020, 20 (4), 2452-2459.

(25) Huang, B.; Clark, G.; Klein, D. R.; Macneill, D.; Navarromoratalla, E.; Seyler, K. L.; Wilson, N.; Mcguire, M. A.; Cobden, D. H.; Xiao, D.; Yao, W.; Jarillo-herrero, P.; Xu, X. Electrical Control of 2D Magnetism in Bilayer $\mathrm{CrI}_{3}$. Nat. Nanotechnol. 2018, 13, 544-548.

(26) Thiel, L.; Wang, Z.; Tschudin, M. A.; Rohner, D.; GutiérrezLezama, I.; Ubrig, N.; Gibertini, M.; Giannini, E.; Morpurgo, A. F.; Maletinsky, P. Probing Magnetism in 2D Materials at the Nanoscale with Single-Spin Microscopy. Science 2019, 364 (6444), 973-976.

(27) Chu, H.; Roh, C. J.; Island, J. O.; Li, C.; Lee, S.; Chen, J.; Park, J. G.; Young, A. F.; Lee, J. S.; Hsieh, D. Linear Magnetoelectric Phase in Ultrathin $\mathrm{MnPS}_{3}$ Probed by Optical Second Harmonic Generation. Phys. Rev. Lett. 2020, 124 (2), 27601.

(28) Šš̌kins, M.; Lee, M.; Mañas-Valero, S.; Coronado, E.; Blanter, Y. M.; van der Zant, H. S. J.; Steeneken, P. G. Magnetic and Electronic Phase Transitions Probed by Nanomechanical Resonators. Nat. Commun. 2020, 11 (1), 2698.

(29) Šškins, M.; Sokolovskaya, E.; Lee, M.; Mañas-Valero, S.; Davidovikj, D.; van der Zant, H. S. J.; Steeneken, P. G. Tunable Strong Coupling of Mechanical Resonance between Spatially Separated $\mathrm{FePS}_{3}$ Nanodrums 2021, 1-22 (arXiv:2107.01262).

(30) Boix-Constant, C.; Mañas-Valero, S.; Córdoba, R.; Coronado, E. Van Der Waals Heterostructures Based on Atomically-Thin Superconductors. Adv. Electron. Mater. 2021, 7, 2000987.

(31) Šškins, M.; Kurdi, S.; Lee, M.; Slotboom, B. J. M.; Xing, W.; Mañas-Valero, S.; Coronado, E.; Jia, S.; Han, W.; van der Sar, T.; van der Zant, H. S. J.; Steeneken, P. G. Nanomechanical Probing and Strain Tuning of the Curie Temperature in Suspended $\mathrm{Cr}_{2} \mathrm{Ge}_{2} \mathrm{Te}_{6}$ Heterostructures. 19th Apr. 2021, arXiv, Condensed Matter; Mesoscale and Nanoscale Physics, arXiv:2104.09614 (accessed 12th Oct. 2021). 